\title{
Effects of chemical composition and B2 order on phonons in bcc Fe-Co alloys
}

\author{
M. S. Lucas, ${ }^{1,2, a)}$ J. A. Muñoz, ${ }^{3}$ L. Mauger, ${ }^{3}$ Chen W. Li, ${ }_{4}^{3}$ A. O. Sheets, ${ }_{5}^{1}$ Z. Turgut, ${ }^{1}$ \\ J. Horwath, ${ }^{1}$ D. L. Abernathy, ${ }^{4}$ M. B. Stone, ${ }^{4}$ O. Delaire, ${ }^{4}$ Yuming Xiao, ${ }^{5}$ and B. Fultz ${ }^{3}$ \\ ${ }^{1}$ Air Force Research Laboratory, Wright-Patterson AFB, Ohio 45433, USA \\ ${ }^{2}$ UTC Inc., 1270 North Fairfield Road, Dayton, Ohio 45432, USA \\ ${ }^{3}$ W. M. Keck Laboratory, California Institute of Technology, 138-78, Pasadena, California 91125, USA \\ ${ }^{4}$ Oak Ridge National Laboratory, 1, Bethel Valley Road, Oak Ridge, Tennessee 37831, USA \\ ${ }^{5}$ HPCAT, Geophysical Laboratory, Carnegie Institution of Washington, 9700 South Cass Ave., Bldg. 434E, \\ Argonne, IL 60439, USA
}

(Received 5 April 2010; accepted 24 May 2010; published online 29 July 2010; corrected 6 August 2010)

The phonon density of states (DOS) gives insight into interatomic forces and provides the vibrational entropy, making it a key thermodynamic function for understanding alloy phase transformations. Nuclear resonant inelastic x-ray scattering and inelastic neutron scattering were used to measure the chemical dependence of the DOS of bcc Fe-Co alloys. For the equiatomic alloy, the $A 2 \rightarrow B 2$ (chemically disordered $\rightarrow$ chemically ordered) phase transformation caused measurable changes in the phonon spectrum. The measured change in vibrational entropy upon ordering was $-0.02 \pm 0.02 k_{\mathrm{B}} /$ atom, suggesting that vibrational entropy results in a reduction in the orderdisorder transition temperature by $60 \pm 60 \mathrm{~K}$. The Connolly-Williams cluster inversion method was used to obtain interaction DOS (IDOS) curves that show how point and pair variables altered the phonon DOS of disordered bcc Fe-Co alloys. These IDOS curves accurately captured the change in the phonon DOS and vibrational entropy of the B2 ordering transition. (C) 2010 American Institute of Physics. [doi:10.1063/1.3456500]

\section{INTRODUCTION}

The Fe-Co alloy system is technologically interesting for its magnetic properties. These alloys have high Curie temperatures, saturation magnetizations, and magnetic permeability. ${ }^{1}$ The equiatomic alloy $\mathrm{Fe}_{0.50} \mathrm{Co}_{0.50}$ undergoes a chemical ordering transition at $730{ }^{\circ} \mathrm{C}$, where a lowtemperature, chemically-ordered $\mathrm{B} 2(\mathrm{CsCl}$ prototype) phase transforms to a high temperature, chemically-disordered A2 [body-centered-cubic (bcc)] phase. The A2 phase can be preserved at room temperature by quenching from high temperatures, and varying amounts of B2 long-range or shortrange order can be obtained in quenched material by controlling the annealing temperatures, ${ }^{2,3}$ or with small alloying additions. ${ }^{4,5} \mathrm{~A}$ drastic change in mechanical properties accompanies the $A 2 \rightarrow B 2$ transition-the ordered phase is brittle, often limiting its use in engineering service. Controlling the state of chemical order is an important strategy for controlling brittleness.

Thermodynamic models of chemical order-disorder transitions date back to work by Bragg and Williams ${ }^{6}$ in $\mathrm{Cu}_{3} \mathrm{Au}$. In their model the enthalpy depends on the numbers of different bonds, and the model includes a configurational entropy that depends on the number of atoms on the two sublattices of the ordered structure. With the cluster variation $\operatorname{method}^{7}$ this configurational entropy has been refined to account for various degrees of short-range order. Only recently however, has it become clear that vibrational entropy can make a thermodynamically-significant contribution to chemi-

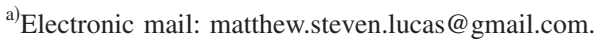

cal order-disorder transitions. ${ }^{8,9}$ In general, it is expected that alloys with large enthalpies of ordering ought to have large changes in vibrational entropy. The stronger, and likely stiffer, chemical bonds of unlike pairs of atoms in the ordered alloy are expected to raise the phonon frequencies, and hence reduce the vibrational entropy. If the change in bond strength and stiffness scales with the critical temperature of ordering, FeCo should have a change in vibrational entropy upon ordering that exceeds the $0.15 k_{\mathrm{B}}$ /atom measured for $\mathrm{Cu}_{3} \mathrm{Au} .{ }^{9}$ Nevertheless, especially because $\mathrm{Fe}-\mathrm{Co}$ alloys are ferromagnetic, we may expect changes in the electron density at the Fermi level with composition or with the state of chemical order. Such changes may alter the electron screening of atom displacements, altering the individual bond stiffnesses and making for a more complex behavior.

To understand the vibrational entropy of alloying and ordering in $\mathrm{Fe}-\mathrm{Co}$ alloys, we undertook the present investigation to measure the phonon density of states (DOS). The experiments used both inelastic neutron scattering (INS) and the technique of nuclear resonant inelastic x-ray scattering (NRIXS). We then assessed how the interatomic forces depend on local chemical arrangements in the alloys by using the cluster expansion method and the cluster inversion method of Connolly and Williams. ${ }^{10}$ This method is usually used to determine the properties of chemically-disordered phases from properties of chemically-ordered phases. There has been recent success, however, in using this inversion method to understand the phonon DOS of ordered thin-film structures of $\mathrm{Fe}-\mathrm{Cr}$ alloys using phonon data from random solid solutions. ${ }^{11}$ For $\mathrm{Fe}-\mathrm{Co}$, we found that the cluster analysis method was successful in predicting changes in the pho- 
TABLE I. Measured lattice parameters $a$, average phonon energies $\langle E\rangle$, and vibrational entropies $S_{\text {vib }}$ of Fe-Co alloys at $300 \mathrm{~K}$ from x-ray diffraction and NRIXS. Values in parentheses are from INS measurements.

\begin{tabular}{lccc}
\hline \hline Sample (nominal) & $\begin{array}{c}a \pm 0.0005 \\
(\mathrm{~nm})\end{array}$ & $\begin{array}{c}\langle E\rangle \pm 0.2 \\
(\mathrm{meV})\end{array}$ & $\begin{array}{c}S_{\text {vib }} \pm 0.02 \\
\left(k_{\mathrm{B}} / \text { atom }\right)\end{array}$ \\
\hline $\mathrm{Fe}$ & 2.8665 & $26.6(26.5)$ & $3.18(3.20)$ \\
$\mathrm{Fe}_{0.90} \mathrm{Co}_{0.10}$ & 2.8686 & 26.4 & 3.21 \\
$\mathrm{Fe}_{0.80} \mathrm{Co}_{0.20}$ & 2.8685 & 26.7 & 3.18 \\
$\mathrm{Fe}_{0.70} \mathrm{Co}_{0.30}$ & 2.8654 & 26.7 & 3.18 \\
$\mathrm{Fe}_{0.60} \mathrm{Co}_{0.40}$ & 2.8592 & 26.8 & 3.18 \\
$\mathrm{Fe}_{0.50} \mathrm{Co}_{0.50} \mathrm{~A} 2$ & 2.8569 & $26.7(26.7)$ & $3.20(3.17)$ \\
$\mathrm{Fe}_{0.50} \mathrm{Co}_{0.50} \mathrm{~B} 2$ & 2.8589 & $26.6(26.7)$ & $3.18(3.16)$ \\
$\mathrm{Fe}_{0.40} \mathrm{Co}_{0.60}$ & 2.8512 & 26.2 & 3.25 \\
$\mathrm{Fe}_{0.30} \mathrm{Co}_{0.70}$ & 2.8469 & $26.1(25.5)$ & $3.27(3.32)$ \\
\hline \hline
\end{tabular}

non DOS upon ordering in $\mathrm{Fe}_{0.50} \mathrm{Co}_{0.50}$ at $300 \mathrm{~K}$. The changes in vibrational entropy with alloying and ordering proved to be small, approximately an order of magnitude smaller than the configurational entropy of ordering. Nevertheless, upon increasing the concentration of Co atoms and Co-Co pairs there was a measurable softening of the low transverse acoustic phonon branch in Fe-Co alloys near the equiatomic composition.

\section{EXPERIMENT AND RESULTS}

Alloys of stoichiometric $\mathrm{Fe}_{1-x_{\mathrm{Co}}} \mathrm{Co}_{x_{\mathrm{Co}}}$ with nominal compositions $\quad x_{\mathrm{Co}}=\{0.70,0.60,0.50,0.40,0.30,0.20,0.10,0\}$ were prepared from shots of $99.99 \%$ natural $\mathrm{Fe}, 96.06 \%$ enriched ${ }^{57} \mathrm{Fe}$, and $99.9+\%$ Co by arc-melting under an argon atmosphere. There was negligible mass loss and little visible surface oxidation after melting. Electron microprobe measurements confirmed the compositions to be accurate to 0.5 at. \%. All of the samples contained between 30 and 40 at. \% ${ }^{57} \mathrm{Fe}$. The ingots were cold rolled to thicknesses between 20 and $50 \mu \mathrm{m}$. X-ray diffractometry showed all samples to be single-phase bcc material with the "cube on edge" texture (a tendency for $\langle 110\rangle$ directions to be normal to the plane of rolling). To reduce the preferred orientation and remove strains, the foils were sealed in quartz tubes, annealed, and quenched. All samples were annealed at temperatures between 900 and $1000{ }^{\circ} \mathrm{C}$ for 2 to $14 \mathrm{~h}$. Longer annealing times were used when lower temperatures were required to avoid the high temperature fcc phase shown on the phase diagram. ${ }^{12}$ To create $\mathrm{B} 2$ order, the as-rolled $\mathrm{Fe}_{0.50} \mathrm{Co}_{0.50}$ alloy was sealed in a quartz tube and annealed at $1000{ }^{\circ} \mathrm{C}$ for $1 \mathrm{~h}$, slowly cooled to $650{ }^{\circ} \mathrm{C}$ over a $24 \mathrm{~h}$ period, and annealed at $600{ }^{\circ} \mathrm{C}$ for four days followed by air cooling. X-ray diffraction patterns acquired after the heat treatments showed no signs of oxidation or preferred orientation. The lattice parameter was found to decrease approximately linearly with increasing cobalt concentration (Table I) in good agreement with the results of Ohnuma et al. ${ }^{12}$ The expansion with ordering is consistent with graphed data of Clegg and Buckley, ${ }^{13}$ although their text states a larger value.

NRIXS (Refs. 14-17) was performed at Beamline 16ID-D at the Advanced Photon Source of the Argonne National Laboratory. The incident photon energy was tuned to $14.413 \mathrm{keV}$, the nuclear resonance energy of ${ }^{57} \mathrm{Fe}$. The

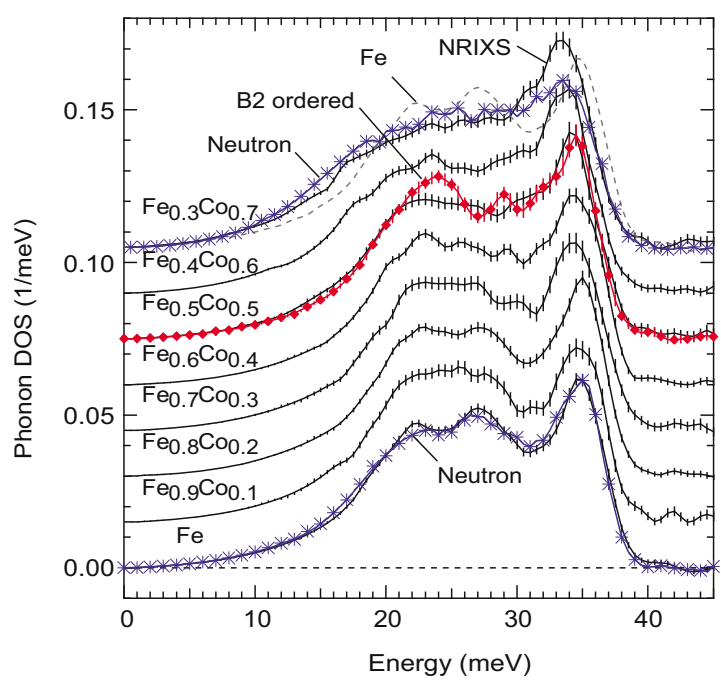

FIG. 1. (Color online) ${ }^{57} \mathrm{Fe}$ partial DOS curves from NRIXS spectra at 300 $\mathrm{K}$. Random solid solutions are shown as solid lines; the B2 ordered alloy by the line with solid diamonds. For comparison to the NRIXS results, the results of INS are shown for $\mathrm{Fe}$ and $\mathrm{Fe}_{0.30} \mathrm{Co}_{0.70}$ as lines with asterisks. Curves are offset by multiples of $0.015 \mathrm{meV}^{-1}$.

NRIXS signal was measured with two avalanche photodiode detectors (APDs) positioned $90^{\circ}$ from the direction of the beam. Each sample was mounted between the two APDs at a grazing angle to the incident photon beam. Data were collected in scans of incident photon energy from -80 to $+80 \mathrm{meV}$ around the resonant energy. The monochromator resolution function was measured in situ using a single APD in the forward direction, and was found to be $2.2 \mathrm{meV}$. Details of the data collection and reduction are described elsewhere. ${ }^{14-17}$ The resulting ${ }^{57} \mathrm{Fe}$ phonon partial DOS (PDOS) curves are shown in Fig. 1. Our NRIXS result for $\mathrm{Fe}_{0.30} \mathrm{Co}_{0.70}$ is consistent with recent NRIXS results on metastable bcc $\mathrm{Fe}_{0.20} \mathrm{Co}_{0.80}$ and $\mathrm{Fe}_{0.10} \mathrm{Co}_{0.90}$ thin films of Laenens et al. ${ }^{18}$

INS measurements on alloys of compositions $x_{\mathrm{Co}}$ $=\{0.70,0.50,0\}$ were performed with the wide angular-range chopper spectrometer (ARCS) at the Spallation Neutron Source at the Oak Ridge National Laboratory. These samples were prepared from natural $\mathrm{Fe}$ (i.e., with no enrichment of ${ }^{57} \mathrm{Fe}$ ) and were sectioned from the cast ingot into pieces of 1 mm thickness using a diamond saw. The INS samples were given the same heat treatments used for the NRIXS samples. Electron microprobe measurements confirmed the compositions of these samples for INS to be accurate to 0.3 at. $\%$. The samples were mounted in thin aluminum foil and held in place by a window covered in neutron absorbing cadmium. The flat plate geometry was placed at a $45^{\circ}$ angle with respect to the incident beam to suppress self shielding. The measurements were performed with a monochromatic beam of neutrons with an incident energy of $80 \mathrm{meV}$. Details of the data collection and reduction procedures are described elsewhere. ${ }^{19-21}$ The resulting neutron-weighted phonon DOS curves are shown in Figs. 1 and 2(b).

Integrating the neutron scattering intensity around the elastic peak from -5 to $+5 \mathrm{meV}$ provides diffraction patterns that are consistent with prior results, including the early work by Shull and Siegel. ${ }^{22}$ For $\mathrm{B} 2 \mathrm{Fe}_{0.50} \mathrm{Co}_{0.50}$, an order 

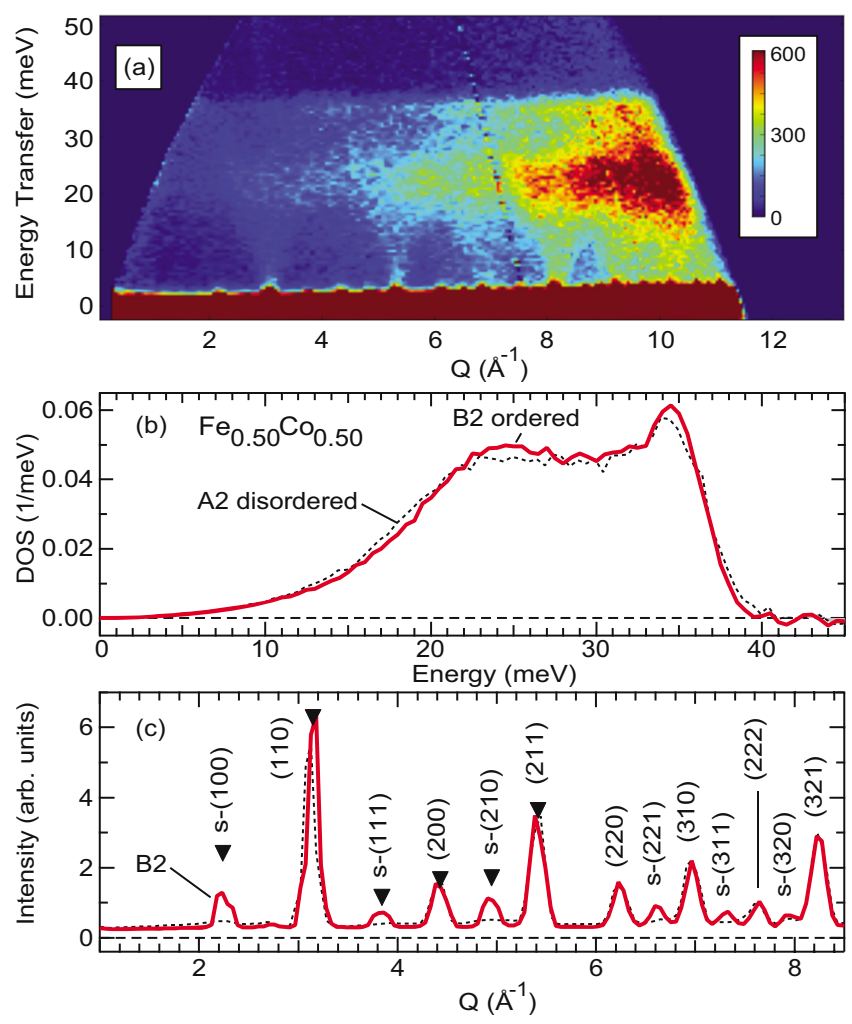

FIG. 2. (Color online) Results of INS on $\mathrm{Fe}_{0.50} \mathrm{Co}_{0.50}$. (a) Intensity as a function of energy transfer and momentum transfer for the B2 ordered sample. (b) Phonon DOS curves for the A2 disordered (dashed line) and B2 ordered (solid line) alloys. (c) Intensity as a function of momentum transfer from the elastic regime of the neutron scattering data, showing the indexed neutron diffraction patterns. The superlattice peaks are labeled with an "s." The downward arrows are the calculated intensities for perfect B2 order $(L=1)$.

parameter $L=0.76$ was determined from the ratio of the intensities of the (100) superlattice peak to the (110) fundamental peak, following the method of Ziller et al. ${ }^{23}$ The intensity of perfectly ordered B2 FeCo was calculated using the Philips X'PERT PLUS software. After successfully matching the calculated intensity to the fundamental peaks in the experimental data, the measured (100) superlattice peaks were found to be weaker than calculated in the ratio $I_{\text {meas }}^{100} / I_{\text {calc }}^{100} \simeq 0.5$. We obtain $L \sim \sqrt{0.5}$, approximately consistent with the result from the method of Ziller et al.

Figure 1 shows that with alloying there is a gradual change in the DOS. Comparison of the $\mathrm{Fe}_{0.30} \mathrm{Co}_{0.70}$ spectrum with that of pure $\mathrm{Fe}$ shows a decrease in the high energy transverse peak of $\sim 1.5 \mathrm{meV}$ and a decrease in the maximum phonon energy of $1 \mathrm{meV}$. The $\mathrm{Fe}_{0.30} \mathrm{Co}_{0.70}$ sample also has a greater number of modes below $19 \mathrm{meV}$. Low energy modes are the most important for vibrational entropy, especially at low temperatures, so the Co-rich alloys have the largest vibrational entropy.

Chemical ordering causes an increase in the number of phonon modes in the energy ranges $21-26 \mathrm{meV}$ and 28-29.5 $\mathrm{meV}$. A measurable decrease in the number of modes between 10 and $20 \mathrm{meV}$ and in the ranges $26.0-27.5 \mathrm{meV}$ and $29.5-34.5 \mathrm{meV}$ is also observed. The maximum phonon energy and first moment of the phonon energy $\langle E\rangle$ is unchanged between the A2 and B2 samples, however.

\section{ANALYSIS}

Comparing the phonon energies in Table I from NRIXS and INS measurements, we see that they are identical for most compositions. Likewise, the neutron-weighted phonon DOS (INS) and phonon partial DOS (NRIXS) curves are quite similar, although differences are seen for the alloy $\mathrm{Fe}_{0.30} \mathrm{Co}_{0.70}$ in Fig. 1. The differences between the two techniques arise from the differences in partial phonon DOSs of $\mathrm{Fe}$ and Co atoms. The NRIXS method measures only the phonon partial DOS of Fe. The INS method measures contributions from the phonon partial DOS of $\mathrm{Fe}$ and that of Co but these contributions are not weighted equally. The efficiency of phonon scattering by neutrons is proportional to the ratio of the total neutron scattering cross-section to the atomic weight. The ratio of phonon scattering efficiencies of Fe to Co is approximately 3 to 1 . For $\mathrm{Fe}_{0.30} \mathrm{Co}_{0.70}$ the difference between the NRIXS and INS results indicate that the phonon partial DOS for Co is softer (i.e., lower energy) than for Fe. Nevertheless, a proper neutron weight correction of the INS results with the NRIXS results gave a Co PDOS that was not very different from the Fe PDOS, with an average phonon energy for the Co modes of $24.7 \mathrm{meV}$. This effect is not large, as also found by Laenens, et al. ${ }^{18}$ for Co-rich alloys, so in what follows we assume the Fe and Co partial DOS curves are the same. Nevertheless, we are careful to compare results from DOS curves obtained from the same technique so that errors are minimized.

\section{A. Vibrational entropy}

The vibrational entropy $S_{\text {vib }}$ is obtained from the normalized phonon DOS, $g(E)$ (Ref. 24):

$$
\begin{aligned}
S_{\mathrm{vib}}(T)= & 3 k_{\mathrm{B}} \int_{0}^{\infty} g(E)([1+n(T)] \ln [1+n(T)] \\
& -n(T) \ln n(T)) d E,
\end{aligned}
$$

where $n(T)=\left\{\exp \left[E /\left(k_{\mathrm{B}} T\right)\right]-1\right\}^{-1}$ is the Planck distribution for phonon occupancy. Table I lists the values of $S_{\text {vib }}$ measured in this experiment. Evaluating Eq. (1) at $300 \mathrm{~K}$, the vibrational entropy of ordering is $\Delta S_{\text {vib }}^{\text {ord }}=S_{\text {vib }}^{\mathrm{B} 2}-S_{\text {vib }}^{\mathrm{A} 2}=$ $-0.02 \pm 0.02 k_{\mathrm{B}} /$ atom. Although we observed measurable changes in the DOS upon ordering, the change in the vibrational entropy is small. The integral in Eq. (1) averages over the phonon modes, washing out the details of the DOS. Upon ordering, the decrease in the vibrational entropy due to the decrease in the number of modes between 10 and $20 \mathrm{meV}$ is largely offset by the increase in the vibrational entropy due to the increase in the number of modes between 21 and 26 $\mathrm{meV}$.

\section{B. Cluster expansion}

Cluster expansions can account for, in varying levels of detail, how physical properties depend on the different local chemical environments of atoms in alloys. Interaction functions are associated with the different clusters, and there has been success in describing the phonon DOS function with relatively few terms in the expansion and relatively few interaction DOS (IDOS) curves. These IDOS functions, 
$\left\{\mathcal{G}_{n}(E)\right\}$, can be obtained from alloys of known structure using the Connolly-Williams cluster inversion technique. ${ }^{10} \mathrm{De}-$ tails of the application of this technique to the phonon DOS can be found elsewhere. ${ }^{11,25,26}$

Spin variables $\sigma$ are used for the two species of atoms, where an $\mathrm{Fe}$ atom is assigned the factor $\sigma=+1$, and a $\mathrm{Co}$ atom $\sigma=-1$. A cluster is made by connecting a number of points $n$ on the lattice. For $n=\{0,1,2\}$ the clusters are the empty lattice, point, and pair clusters, respectively. The chemical arrangement is parameterized by the correlation function

$$
\xi_{n}=\frac{1}{N_{n}} \sum_{\left\{p_{i}\right\}} \sigma_{p_{1}} \sigma_{p_{2}} \cdots \sigma_{p_{n}} .
$$

Here $n$ also denotes the order of each term in the expansion, $N_{n}$ is the number of clusters of type $n$, and $\sigma_{p}$ is the value of the spin variable at site $p$. For a random solid solution of Fe and $\mathrm{Co}$ atoms, the correlation function is

$$
\xi_{n}=\left(1-2 x_{\mathrm{Co}}\right)^{n} .
$$

The correlation function matrix $\left[\xi_{m, n}\right]$ describes the local chemical environments in a set of alloys labeled by $m$. Given a set of interaction phonon DOS functions, we approximate the phonon DOS $g_{m}(E)$ of the alloy $m$ as:

$$
g_{m}(E)=\sum_{n=0}^{\nu-1} \mathcal{G}_{n}(E) \xi_{m, n},
$$

where $\nu$ is the number of terms used in the expansion. The IDOS curves $\left\{\mathcal{G}_{n}(E)\right\}$ are obtained by inverting the correlation function matrix

$$
\mathcal{G}_{n}(E)=\sum_{m=0}^{\nu-1} g_{m}(E)\left[\xi_{m, n}\right]^{-1} .
$$

Here a set of $g_{m}(E)$ are measured phonon DOS curves of alloys whose local atomic arrangements and correlation functions are known.

A basic question is how many terms are needed in the cluster expansion to describe adequately the phonon DOS. This choice is simplified by using a least-squares inversion, which is possible because a correlation function matrix for random solid solutions is a Vandermonde matrix. ${ }^{11}$ Before inverting, the DOS functions were rescaled to the maximum phonon energy of the $\mathrm{Fe}_{0.50} \mathrm{Co}_{0.50}$ DOS to ensure proper normalization of the IDOS functions. The least-squares inversion was performed for the alloys $x_{\mathrm{Co}}$ $=\{0.70,0.60,0.40,0.30,0.20,0.10,0\}$. The equiatomic alloy was not included for the inversion. The best fit was obtained using a three term expansion, and the resulting IDOS functions are shown in Fig. 3(a).

The IDOS functions have physical meaning from the type of cluster they represent. The $n=0$ term corresponds to the empty lattice, and the corresponding IDOS $\mathcal{G}_{0}(E)$ is the DOS of a $\mathrm{Fe}_{0.50} \mathrm{Co}_{0.50}$ random solid solution. The function $\mathcal{G}_{0}(E)$ is in very good agreement with the measured A2 $\mathrm{Fe}_{0.50} \mathrm{Co}_{0.50}$ DOS (Fig. 3), considering that the measured $\mathrm{Fe}_{0.50} \mathrm{Co}_{0.50}$ DOS was not used to calculate $\mathcal{G}_{n}(E)$ (owing to the tendency to develop B2 order at this composition). The

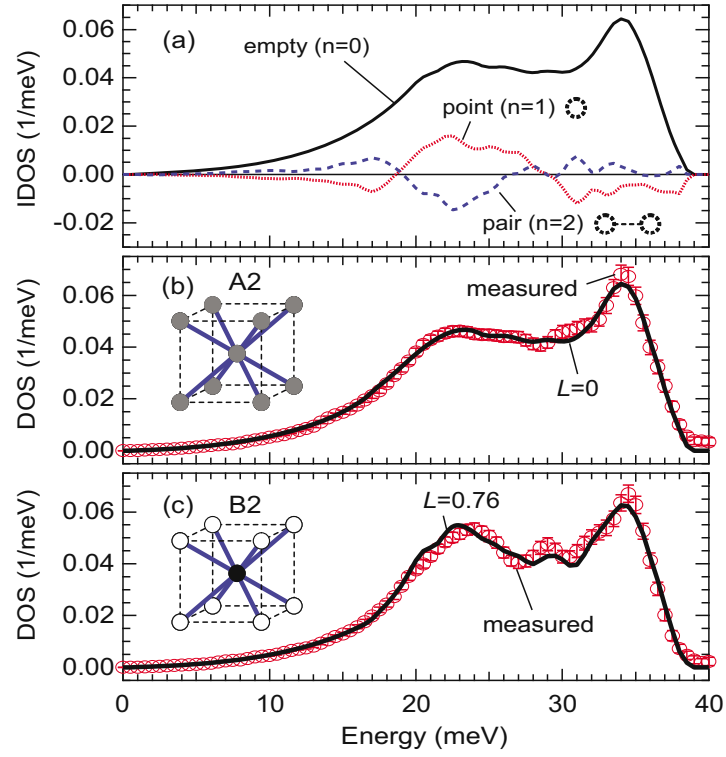

FIG. 3. (Color online) (a) Interaction phonon DOS curves from cluster inversion of random solid solutions of Fe-Co. [(b) and (c)] Comparison of the measured DOS (open markers with error bars) for $\mathrm{Fe}_{0.50} \mathrm{Co}_{0.50}$ to the DOS determined from the cluster expansion (solid line) for A2 $(L=0)$ and B2 $(L=0.76)$ ordering. Inset are diagrams of the following order: Fe atoms (black), Co atoms (white), and 50:50 probability of Fe or Co atom (gray). The thick solid lines indicate first-neighbor pairs and the dashed lines indicate second-neighbor pairs.

$n=1$ term corresponds to the point cluster, and gives the concentration dependence of the DOS. When Fe is added to the equiatomic alloy (making the alloy Fe-rich) the function $\mathcal{G}_{1}(E)$ is added to $\mathcal{G}_{0}(E)$, causing an increase in the number of modes between 19 and $29 \mathrm{meV}$ and a decrease in the numbers of all other modes. When $\mathrm{Co}$ is added to the equiatomic alloy (making the alloy Co-rich) the function $\mathcal{G}_{1}(E)$ is subtracted from $\mathcal{G}_{0}(E)$. The $n=2$ term corresponds to the pair cluster, and gives the pairwise dependence of the DOS. An increase in the number of like pairs ( $\mathrm{Fe}-\mathrm{Fe}$ or $\mathrm{Co}-\mathrm{Co}$ ) causes a decrease in the numbers of modes between 19 and $29 \mathrm{meV}$, and an increase in the numbers of all other modes. Unlike pairs $(\mathrm{Fe}-\mathrm{Co})$ have the opposite effect.

For a fixed composition, the effect of chemical ordering on the phonon DOS is determined only by $\mathcal{G}_{2}(E)$, which describes the effects of first-neighbor pairs. With B2 ordering, there is a decrease in the number of like pairs and the shape of the phonon DOS curve for a disordered $\mathrm{Fe}_{0.50} \mathrm{Co}_{0.50}$ alloy should change by subtracting the function $\mathcal{G}_{2}(E)$ from the disordered phonon DOS [which is the function $\mathcal{G}_{0}(E)$ in Fig. 3(a)]. The amount to be subtracted depends on the order parameter $L$. For the equiatomic alloy, the average fraction of unlike pairs ${ }^{27}$ is $\left(L^{2}+1\right) / 2$. Following the prescription of Eq. (2), the pair correlation function for the equiatomic alloy with an arbitrary degree of order is $\xi_{2}=-L^{2}$. Our diffraction measurements indicated $L=0.76$ for the partially-ordered alloy, for which we obtain the correlation function $\xi_{2}=-0.58$ (and $\xi_{2}=0$ for the disordered alloy). Using Eq. (4) with this $\xi_{2}=-0.58$ and the $\mathcal{G}_{2}(E)$ function obtained from the set of disordered $\mathrm{Fe}-\mathrm{Co}$ alloys, we obtain the result presented in Fig. 3(c). The overall agreement is quite good, showing a bunching in energy of the transverse modes upon ordering. 
Evaluation of Eq. (1) for the IDOS in Fig. 3(a) gives the interaction vibrational entropies $\mathcal{S}_{n}^{\mathrm{vib}}$. We obtain $3.209 k_{\mathrm{B}} /$ atom, $0.012 k_{\mathrm{B}} /$ atom, and $0.026 k_{\mathrm{B}} /$ atom for $n$ $=\{0,1,2\}$, respectively. From a cluster expansion of the alloys (not including the equiatomic alloy) we obtain a vibrational entropy upon ordering of $\Delta S_{\mathrm{vib}}^{\mathrm{ord}}=S_{\mathrm{vib}}^{\mathrm{B} 2}-S_{\mathrm{vib}}^{\mathrm{A} 2}=-\mathcal{S}_{2}^{\mathrm{vib}}=$ $-0.026 k_{\mathrm{B}} /$ atom. For $L=0.78$ we obtain a value of $-0.016 k_{\mathrm{B}} /$ atom. This is in very good agreement with our direct observation of $-0.02 \pm 0.02 k_{\mathrm{B}}$ /atom.

\section{DISCUSSION}

In alloys that develop chemical order, unlike bonds are stronger than like bonds. We generally expect the overall bond stiffness to increase as ordering produces more unlike bonds. Stiffer bonds decrease the vibrational entropy of the ordered phase, destabilizing it at higher temperatures and causing a reduction in the critical temperature for ordering. This simple analysis is usually true for ordered alloys, ${ }^{9}$ and ordering in $\mathrm{FeCo}$ is no exception. Using the phonon DOS curves measured for our samples that were largely ordered and disordered, we find $\Delta S_{\text {vib }}(300 \mathrm{~K})=-0.02 \pm 0.02 k_{\mathrm{B}} /$ atom, where the ordered alloy has a slightly smaller vibrational entropy than the disordered alloy. This value was assessed at $300 \mathrm{~K}$, and it may differ at higher temperatures owing to anharmonic effects.

Alloying and ordering cause only small effects on the phonons and hence the vibrational entropy of bcc Fe-Co alloys. Trends are nevertheless observable, and can be understood in terms of changes in the chemical bonds. The average phonon energy, and the phonon DOS itself, show little change over composition range from pure $\mathrm{Fe}$ to $\mathrm{Fe}_{0.50} \mathrm{Co}_{0.50}$. At higher Co concentrations there is a reduction in phonon stiffness. This phonon softening occurs after the peak in the Slater-Pauling curve of magnetization versus electron concentration, which has been the subject of numerous studies. Phonon softening is expected if there is increased screening of atom displacements caused by a higher DOS at the Fermi level. Such an increase in the electron DOS does occur beyond the peak of the Slater-Pauling curve when the Fermi level moves out of a valley in the electronic DOS of the minority spin electrons. ${ }^{28}$

The average effect of Co concentration, as quantified by $\mathcal{G}_{1}(E)$, is to spread apart the frequencies of the transverse modes, and this effect is observably strongest at Co concentrations exceeding 50 at. \% Co where $\mathcal{G}_{2}(E)$ tends to make a similar contribution. (The contributions $\mathcal{G}_{1}(E)$ and $\mathcal{G}_{2}(E)$ tend to cancel for concentrations below $50 \% \mathrm{Co}$, so the $\mathcal{G}_{0}(E)$ function describes the phonon DOS fairly well for Fe-rich alloys.)

Our results show that chemical ordering causes a shift to higher energies of the transverse modes. In pure Fe, Satija et $a l^{29}$ reported a substantial decrease in the energy of the $[110] \mathrm{T}_{2}$ mode at reciprocal point $(1.5,0.5,0)$ with increasing temperature. The energy of this mode decreases from $\sim 19.5 \mathrm{meV}$ at $300 \mathrm{~K}$ to $\sim 11.5 \mathrm{meV}$ at the Curie temperature. This shows a large dependence of the transverse modes with the saturation magnetization through the increase in temperature. For FeCo there is an increase in the saturation moment of $3 \%$ upon ordering. ${ }^{30}$ It is reasonable to expect a stiffening of the transverse modes upon ordering in FeCo due to this magnetoelastic coupling.

Ordering is usually accompanied by a decrease in specific volume. From the results of Table I we see that ordering in $\mathrm{FeCo}$ is an exception, with an expansion of lattice parameter of approximately $0.05 \%$ upon ordering. Assuming a Grüneisen parameter $\gamma=2$, phonon frequencies are expected to decrease upon ordering by a fraction of approximately $\gamma 3 \Delta a / a \simeq 3 \times 10^{-3}$, causing an increase in vibrational entropy of approximately $0.01 \mathrm{k}_{\mathrm{B}}$ /atom. ${ }^{9}$ Evidently this is not the dominant effect. Our measurements of the phonon DOS show a slight average stiffening with ordering, giving a vibrational entropy of ordering of $-0.02 \pm 0.02 k_{\mathrm{B}} /$ atom at 300 $\mathrm{K}$. For comparison, the corresponding change in configurational entropy upon ordering is given by

$$
\Delta S_{\mathrm{cf}}=-\frac{k_{\mathrm{B}}}{2}[(1+L) \ln (1+L)+(1-L) \ln (1-L)],
$$

which is $-0.33 k_{\mathrm{B}} /$ atom for $L=0.76$. The contribution of the vibrational entropy to the critical temperature is

$$
\Delta T_{\text {crit }}^{\mathrm{vib}}=-\frac{\Delta S_{\mathrm{vib}}}{\Delta S_{\mathrm{cf}}} T_{\text {crit }},
$$

so the vibrational entropy acts to stabilize the disordered phase, reducing the critical temperature by $60 \pm 60 \mathrm{~K}$ if the phonon DOS curves change similarly with temperature.

\section{CONCLUSIONS}

Phonon spectra were measured for bcc Fe-Co alloys by NRIXS and by INS. The ${ }^{57} \mathrm{Fe}$ phonon partial DOS curves from NRIXS were quite similar to the neutron-weighted phonon spectra from INS, so the phonon partial DOS of Co was generally similar to that of Fe, although somewhat lower in frequency. The phonon DOS of disordered $\mathrm{Fe}-\mathrm{Co}$ alloys underwent little change from compositions of pure $\mathrm{Fe}$ to $\mathrm{Fe}_{0.50} \mathrm{Co}_{0.50}$ but softened at higher Co concentrations (after the peak in magnetization of the Slater-Pauling curve). A three-term cluster expansion analysis of the disordered solid solutions gave interaction phonon DOS curves that showed Co broadened the energy spread of the transverse phonon branches, referenced to the alloy $\mathrm{Fe}_{0.50} \mathrm{Co}_{0.50}$. The development of chemical B2 order in the alloy $\mathrm{Fe}_{0.50} \mathrm{Co}_{0.50}$ narrowed the energy spread of the transverse phonon branches but had little effect on the average phonon frequencies. The change in phonon DOS upon ordering was used to calculate a small change in vibrational entropy upon ordering, only $-0.02 \pm 0.02 k_{\mathrm{B}} /$ atom at $300 \mathrm{~K}$. This would suggest that the critical temperature of ordering would be lowered by $60 \pm 60 \mathrm{~K}$ due to vibrational entropy. Nevertheless, the change in phonon DOS upon ordering could be obtained reliably from the IDOS curves obtained from the cluster expansion analysis of the disordered solid solutions of Fe-Co.

\section{ACKNOWLEDGMENTS}

The portions of this work conducted at Oak Ridge National Laboratory were supported by the Scientific User Facilities Division and by the Division of Materials Sciences 
and Engineering, Office of Basic Energy Sciences, DOE. This work was supported by the Department of Energy through the Basic Energy Sciences Grant Nos. DE-FG0203ER46055 and BES-MS, W-31-109-ENG-38. Portions of this work were performed at HPCAT (Sector 16), Advanced Photon Source (APS), Argonne National Laboratory. Use of the HPCAT facility was supported by DOE-BES, DOENNSA (CDAC), NSF, DOD-TACOM, and the W. M. Keck Foundation. Use of the APS was supported by DOE-BES under Contract No. DE-AC02-06CH11357. This work benefited from DANSE software developed under NSF Grant No. DMR-0520547.

${ }^{1}$ T. Sourmail, Prog. Mater. Sci. 50, 816 (2005).

${ }^{2}$ B. Fultz, Acta Metall. 37, 823 (1989).

${ }^{3}$ B. Fultz, Phys. Rev. B 44, 9805 (1991).

${ }^{4}$ J. H. White and C. V. Wahl, U.S. Patent No. 1,862,559, (June 14, 1932)

${ }^{5}$ B. Fultz, H. H. Hamdeh, and D. H. Pearson, Acta Metall. 37, 2841 (1989).

${ }^{6}$ W. L. Bragg and E. J. Williams, Proc. R. Soc. London, Ser. A 145, 699 (1934).

${ }^{7}$ R. Kikuchi, Phys. Rev. 81, 988 (1951).

${ }^{8}$ L. Anthony, L. J. Nagel, J. K. Okamoto, and B. Fultz, Phys. Rev. Lett. 73, 3034 (1994).

${ }^{9}$ B. Fultz, Prog. Mater. Sci. 55, 247 (2010).

${ }^{10}$ J. W. D. Connolly and A. R. Williams, Phys. Rev. B 27, 5169 (1983).

${ }^{11}$ M. S. Lucas, A. Papandrew, B. Fultz, and M. Y. Hu, Phys. Rev. B 75 , 054307 (2007).

${ }^{12}$ I. Ohnuma, H. Enoki, O. Ikeda, R. Kainuma, H. Ohtani, B. Sundman, and
K. Ishida, Acta Mater. 50, 379 (2002).

${ }^{13}$ D. W. Clegg and R. A. Buckley, Met. Sci. 7, 48 (1973).

${ }^{14}$ E. E. Alp, W. Sturhahn, T. S. Toellner, J. Zhao, M. Hu, and D. E. Brown, Hyperfine Interact. 144-145, 3 (2002).

${ }^{15}$ M. Seto, Y. Yoda, S. Kikuta, X. W. Zhang, and M. Ando, Phys. Rev. Lett. 74, 3828 (1995).

${ }^{16}$ W. Sturhahn, T. S. Toellner, E. E. Alp, X. Zhang, M. Ando, Y. Yoda, S. Kikuta, M. Seto, C. W. Kimball, and B. Dabrowski, Phys. Rev. Lett. 74, 3832 (1995)

${ }^{17}$ W. Sturhahn, Hyperfine Interact. 125, 149 (2000)

${ }^{18}$ B. Laenens, N. Planckaert, M. Sternik, P. T. Jochym, K. Parliński, A. Vantomme, and J. Meersschaut, Phys. Rev. B 79, 224303 (2009).

${ }^{19}$ M. Kresch, Ph.D. thesis, California Institute of Technology, 2009.

${ }^{20}$ B. Fultz, T. Kelley, J. Lin, J. Lee, O. Delaire, M. Kresch, M. McKerns, and M. Aivazis, "Experimental Inelastic Neutron Scattering," http:// drchops.caltech.edu/Docs, (2009).

${ }^{21}$ M. Kresch, O. Delaire, R. Stevens, J. Y. Y. Lin, and B. Fultz, Phys. Rev. B 75, 104301 (2007).

${ }^{22}$ C. G. Shull and S. Siegel, Phys. Rev. 75, 1008 (1949).

${ }^{23}$ T. Ziller, G. Le Caër, O. Isnard, P. Cénédèse, and B. Fultz, Phys. Rev. B 65, 024204 (2001).

${ }^{24}$ D. C. Wallace, Statistical Physics of Crystals and Liquids (World Scientific, Singapore, 2002)

${ }^{25}$ M. S. Lucas, M. Kresch, R. Stevens, and B. Fultz, Phys. Rev. B 77, 184303 (2008).

${ }^{26}$ M. S. Lucas, Ph.D. thesis, California Institute of Technology, 2008.

${ }^{27}$ L. A. Girifalco, Statistical Mechanics of Solids (Oxford University Press, New York, 2000).

${ }^{28}$ R. H. Victora and L. M. Falicov, Phys. Rev. B 30, 259 (1984).

${ }^{29}$ S. K. Satija, R. P. Comés, and G. Shirane, Phys. Rev. B 32, 3309 (1985).

${ }^{30}$ D. I. Bardos, J. Appl. Phys. 40, 1371 (1969). 